Human Ethology Bulletin 31 (2016)2: 45-51

Brief Report

\title{
AN EVOLUTIONARY VIEW ON CONFLICT ESCALATION IN TEAM SPORTS COMPETITIONS
}

\author{
Cyril C. Grueter, Liam Johnson, Michael Leaning, Ming Jay Tan, Zane Jones \& Jeremy \\ Peris
}

School of Anatomy, Physiology and Human Biology, University of Western Australia, Perth, Australia

cyril.grueter@uwa.edu.au

\begin{abstract}
It has been theorized that escalated aggression in animal intergroup contests is more likely to occur when the fighting ability or resource holding potential (RHP) of the two involved groups are similar. In an attempt to test this model for human intergroup contests, we investigated the effect of differences in RHP on aggression during ice hockey games. We mined data on penalty minutes accrued and differences in team ranks for professional ice hockey games $(n=1230)$ in the National Hockey League of Northern America (NHL). The former served as a surrogate measure of conflict escalation and the latter was used as a proxy for RHP. Using a linear mixed model approach, we found a significant but weak negative effect of differences in team ranks on penalty minutes, i.e. escalation was marginally more frequent when teams were more similar in rank. Various possible shortcomings of the study design are discussed.
\end{abstract}

Key words: Resource holding potential, conflict escalation, sports contests.

\section{INTRODUCTION}

In dyadic and group-level animal contests, both the relative fighting ability of the contestants and the consequences of winning or losing (i.e. the resource value at stake) are thought to influence the probability of conflict escalation and encounter outcomes (Arnott 
and Elwood, 2008; Enquist and Leimar, 1987; Maynard Smith and Parker, 1976; Sherratt and Mesterton-Gibbons 2013). Animals are expected to receive optimal fitness payoffs if they can reliably assess their opponent's resource holding potential (RHP) relative to their own and thereby avoid physical contests they are likely to lose (Maynard Smith and Parker, 1976; Parker, 1974). Parker (1974) theorized that escalation is more likely to occur when the two contestants have similar RHPs. RHP refers to fighting or competitive ability of opponents (body size, group size, experience etc.) (Arnott and Elwood, 2009). Several empirical studies have documented negative associations between contest duration/escalation and size disparity in conflicts between individuals (e.g. Austad 1983; Jonart et al. 2007).

However, little is known about the applicability of the above model to group-level contests (but see Batchelor and Briffa 2011; Batchelor et al 2011). In the case of intergroup conflict, RHP can be operationally defined as group size, but the individual attributes of the group members may also play a part in determining competitive ability. Van Belle and Scarry (2015) found that black howler monkeys (Alouatta pigra) increased their participation in inter-group interactions when male group sizes were equal. Human intergroup contests can be analysed using a similar conceptual framework. In a study examining human interstate wars, wars lasted less long when there was a large disparity in power (measured by the composite index of national capability) between sides (Briffa 2014). Stulp et al. (2012) recently investigated determinants of conflict escalation in human group contests using football (Bundesliga) and basketball (NBA) data. Their results showed that when competitors differed little in RHP, i.e. were more evenly matched in terms of league ranks, escalation (number of fouls committed) was more likely to occur.

We tested the above assumption using data from a professional sporting platform. Resource holding potential or competitive ability can be approximated by the teams' performance, or rank in the competition, and aggression/conflict escalation as the number of fouls committed (Stulp et al. 2012). We used games played in the National Hockey League of Northern America (NHL) as an example of human group contests. Following from the predictions outlined above, it is anticipated that similarities in teams' RHP (measured by their difference in ranking at the time of play) will result in greater aggression displayed (measured in the number of penalty minutes accrued).

\section{METHOD}

\section{Data collection}

Ice hockey is a contact sport played by two teams of six players on a rectangular ice rink. The teams use hockey sticks to try and push a small disc, the puck, into a goal at the short end of the rink. Traditionally, ice hockey has been known as an aggressive sport. It is one of the few sports that include specific rules allowing players to fight one another separate to the rules of general play fouls. The NHL is the highest level of professional ice hockey in the world. This 
league was chosen as it is known for the occurrence of aggression involving high quality individual athletes.

Statistics from the October 2013 - June 2014 NHL regular season consisting of 30 teams competing in 82 games each (total number of games $=1230$ ) were analyzed. A total of 18 games played during the initial weeks of the season were omitted from the data as not all of the teams had played a game up until this point and therefore not established a ranking within the competition.

Resource holding potential was estimated using team's position on the total league ladder at the time of play (Stulp et al. 2012). The difference in resource holding potential was calculated by subtracting the lower-ranked team's ladder position from the higherranked team's.

Aggression was measured by the number of penalty minutes accrued by each team during a game (controlled for total length of game, as some games went into overtime and to a shootout). Penalty minutes are awarded as punishment for infractions on the field of play. There are two main types of penalties; a minor, which is worth two minutes, and a major, which is worth five minutes. Majors are awarded for more serious infractions of the rules, such as fighting, charging and boarding, than minors, which may be awarded for hooking, kneeing and tripping, amongst others. There are also misconduct penalties, which are awarded due to a combination of a number of minors and majors accrued by the same player. Misconduct penalties result in a ten minute suspension during the game. There are also 'game misconduct' and 'match' penalties that don't result in any minute suspensions, but result in ejections from the game. The NHL charges both of these statistically as ten minutes to the offending player. For the purposes of this study, all statistics will follow those collected by the NHL and penalty minutes are as reported on their website.

\section{Data analysis}

Linear Mixed model fit by maximum likelihood were conducted using the function "lmer" of the lme4 package in $\mathrm{R}$ statistical software 3.1.0 (The $\mathrm{R}$ Foundation for Statistical Computing, Vienna, Austria, http://www.r-project.org). Number of penalty minutes was entered as the response variable in the model and team rank (RHP) was treated as a fixed effect. The identities of home and visiting teams were included as random effects. Number of penalty minutes was log transformed. Parameter-specific p-values were approximated using a normal distribution: coefs $\$ \mathrm{p} . \mathrm{z}<-2^{*}(1-\operatorname{pnorm}(\operatorname{abs}(\operatorname{coefs} \$$ t.value $)))$. For all statistical tests, we set the level of significance at $\mathrm{p} \leq 0.05$.

\section{RESULTS}

There was a slight negative effect of RHP difference on penalty minutes, i.e. escalation was marginally more likely to manifest when teams were more similar in rank (Table 1; Figure $1)$. 
Table 1: Effect of team rank (RHP) on the number of penalty minutes accumulated in a sample of NHL ice hockey games.

\begin{tabular}{lcccc}
\hline & Estimate & Std error & T value & $\mathbf{p}$ \\
\cline { 2 - 5 } Intercept & -0.502028841 & 0.016488987 & -30.446311 & \\
RHP & -0.002834147 & 0.001131973 & -2.503724 & 0.0122894 \\
\hline
\end{tabular}

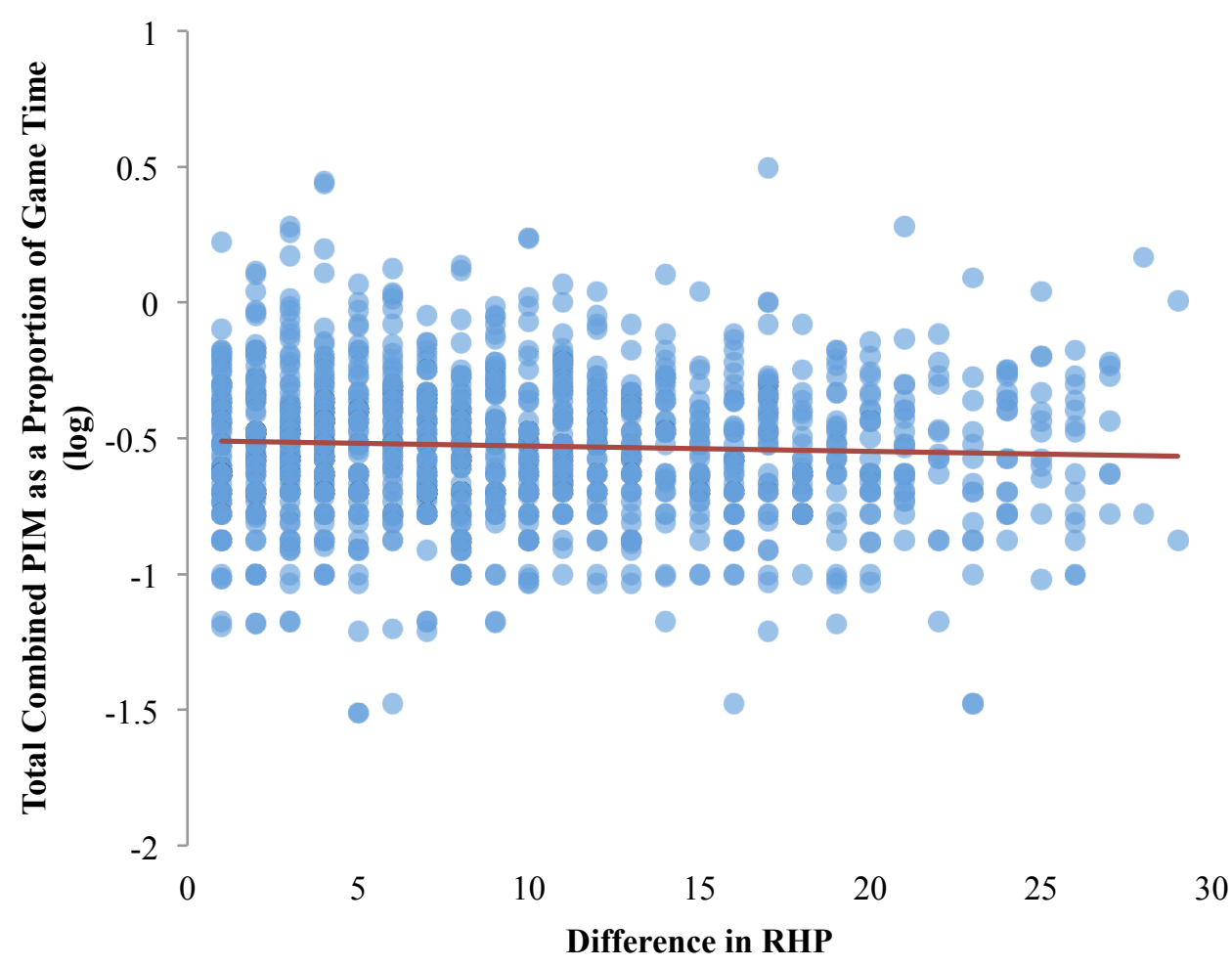

Figure 1: Plot of combined penalties in minutes (PIM) as a proportion of game time (y variable) vs. difference in RHP ( $\mathrm{x}$ variable).

\section{DISCUSSION}

Our findings provide some limited support for Stulp et al.'s (2012) conclusion that in group sports contests escalation is more frequently seen when contestants are more similar in absolute ranks. The effect was in the predicted direction, but the effect size (slope estimate) was low given the relatively large sample size used in the study. Collectively, these findings are consistent with theoretical predictions originating from dyadic animal conflict theory 
that conflicts are more likely to be characterized by higher levels of aggression when the RHP of the contenders are more similar (Parker, 1974).

Since studying sports competitions to shed light on the evolutionary basis of intergroup conflict constitutes a relatively novel and not foolproof approach, we discuss possible shortcomings of the study design and make recommendations for refinements.

Firstly, data on contest derived from ice hockey games may differ qualitatively and quantitatively from other team sports. Ice hockey is considered a full-contact sport where games revolve around a great deal of physical aggression. Sports such as basketball and soccer are considered limited contact sports, where rules and regulations are specifically implemented to prevent physical contact between players, making them vastly different in aggressiveness when compared to ice hockey.

Resource holding potential is oftentimes equated with the size of the groups in the contest (e.g. Wilson et al. 2001). One flaw with choosing league ranking as a proxy for RHP is that accrual of penalty minutes decreases the group size in the competition. Hence, taking into account actual group size would be an essential step for further studies.

Moreover, a potentially more suitable proxy for RHP or fighting ability than league ranking would be the size of individual players on the ice, roles of the players on the ice or a combination of these factors. Size of players would probably represent a suitable indicator of RHP due to the physical nature of aggression in ice hockey; a larger body will elicit more damage on an opponent, the main aim of most shows of aggression in the game. The size of the players on the ice changes throughout the game as coaches make personnel changes and substitutions throughout the game, meaning that a study using size as a proxy for RHP would require researchers to conduct more detailed analyses during games. The roles of the players may indicate RHP of teams as there are players who have the specific job of placing hits on opponents, known as enforcers. However, these are not permanent positions on the ice and the individuals in this role are not the sole demonstrators of aggression. Lastly, the effect of home advantage versus visiting opponents (Courneya and Carron, 1992; Neave and Wolfson, 2003) may also have a bearing on the realized RHP of a team.

We used the number of accrued penalty minutes as a proxy for aggression. Penalty minutes are awarded for breaches of the rules, but these are not all aggressive acts. For example, simulation, where a player makes out he has received an unfair check or hit, is not an aggressive manoeuver, but it can result in penalty minutes being awarded.

Other possible indicators of aggression shown may stem from fights in the NHL. The NHL has rules that allow players to fight during games and specifies specific acts they may and may not perform. The enforcer players are often responsible for starting fights, but there is another role player, the pest, whose job is to goad other players into engaging in a fight. These are two different tactics, but it would be difficult to argue that either does not show aggression from the part of one player involved. In the 2013/2014 NHL regular season, there were 469 fights in 366 games (29.8\% of games) involving 288 players. 78 games involved more than one fight. This is a substantial number and analyses of these fights statistics, the teams and players involved, the length of fights, the outcome of fights and the 
outcome of these games could be used as proxies for aggression shown. Furthermore, ice hockey involves legal hits. The number, force and location of these hits may serve as a proxy for aggression. The best measure of aggression may thus involve a combination of factors and not be limited to aggression shown that is outside the rules of hockey.

Human error may also play a part in the results found in the present study. Different referees officiate each game and although they follow the same rulebook, they have different interpretations of incidents that occur on the ice and make different rulings as a result. For example, one referee may decide the same incident deserves a minor penalty, another may decide it warrants a major and another may not think it warrants a penalty at all. This would affect the consistency of penalty minutes accrued in games. Future studies should control for the effects of inter-individual variation in refereeing styles.

In sum, sport contests may be a promising avenue for exploring the evolutionary nature of intergroup competition and it is hoped that attempts at overcoming some of the limitations identified in the present study will be made in follow-up studies on a variety of sporting disciplines.

\section{REFERENCES}

Arnott, G., \& Elwood, R. W. (2008). Information gathering and decision making about resource value in animal contests. Animal Behaviour 76, 529-542. DOI

Arnott, G., \& Elwood, R. W. (2009). Assessment of fighting ability in animal contests. Animal Behaviour 77, 991-1004. DOI

Austad, S. (1983). A game theoretical interpretation of male combat in the bowl and doily spider (Frontinella pyramitela). Animal Behaviour 31, 59-7. DOI

Batchelor, T., \& Briffa, M. (2011) Fight tactics in wood ants: individuals in smaller groups fight harder but die faster. Proceedings of the Royal Society B 278, 3243-3250. DOI

Batchelor, T. P., Santini, G., Briffa, M. (2012). Size distribution and battles in wood ants: group resource-holding potential is the sum of the individual parts. Animal Behaviour 83, 111-117. $\underline{\text { DOI }}$

Briffa, M. (2014). What determines the duration of war? Insights from assessment strategies in animal contests. PLoS ONE 9, e108491. DOI

Courneya, K. S., \& Carron, A. V. (1992). The home advantage in sport competitions: a literature review. Journal of Sport \& Exercise Psychology 14. DOI

Enquist, M., \& Leimar, O. (1987). Evolution of fighting behaviour: the effect of variation in resource value. Journal of Theoretical Biology 127, 187-206. DOI

Jonart, L. M., Hill, G. E., Badyaev, A. V. (2007). Fighting ability and motivation: determinants of dominance and contest strategies in females of a passerine bird. Animal Behaviour 74, 16751681. DOI 
Maynard Smith, J., \& Parker, G. A. (1976). The logic of asymmetric contests. Animal Behaviour 24, 159-175. DOI

Neave, N., \& Wolfson, S. (2003). Testosterone, territoriality, and the 'home advantage'. Physiology \& Behavior 78, 269-275. DOI

Parker, G. A. (1974). Assessment strategy and the evolution of fighting behaviour. Journal of Theoretical Biology 47, 223-243. DOI

Sherratt, T., \& Mesterton-Gibbons, M. (2013). Models of group or multi-party contests. In: Hardy, I. C. W., \& Briffa, M. (editors): Animal Contests. Cambridge University Press, Cambridge, p. 33-46. DOI

Smith, J. M., \& Parker, G. A. (1976). The logic of asymmetric contests. Animal Behaviour 24, 159175. DOI

Stulp, G., Kordsmeyer, T., Buunk, A. P., Verhulst, S. (2012). Increased aggression during human group contests when competitive ability is more similar. Biology Letters $8,921-923$. DOI

Van Belle, S., \& Scarry, C. J. (2015). Individual participation in intergroup contests is mediated by numerical assessment strategies in black howler and tufted capuchin monkeys. Philosophical Transactions of the Royal Society B. 370, 20150007. DOI

Wilson, M. L., Hauser, M. D., Wrangham, R. W. (2001). Does participation in intergroup conflict depend on numerical assessment, range location, or rank for wild chimpanzees? Animal Behaviour 61, 1203-1216. DOI 